\title{
Synchronization of Underwater Glider with Uncertainties Using Adaptive Fuzzy Sliding Mode Control
}

\author{
Li-Ming Wang \\ Faculty of physics and electronic information \\ Langfang Teachers University \\ Langfang, China \\ E-mail: wlm_shooker@163.com
}

\begin{abstract}
In this paper we study the synchronization of an underwater glider by means of the adaptive fuzzy sliding mode control technology. The sufficient conditions for the synchronization are obtained even though the slave underwater glider is unknown. Based on the Lyapunov stability theorem, the stability the synchronization is proved theoretically. Numerical simulations further support the theoretical results of the paper and demonstrate the efficiency of the proposed methods.
\end{abstract}

Keywords-underwater glider; adaptive fuzzy sliding mode control; synchronization; master-slave system

\section{INTRODUCTION}

Over the past two decades, an underwater glider has been widely used in the marine-environment monitoring, the unenclosed aquaculture and ocean exploration, especially in the long-term, large-scale oceanographic monitoring ${ }^{[1]}$. The UG has a number of attractive features, such as a high efficiency, a low noise, a large range, lower maintenance cost, lower pollution and a compact structure ${ }^{[2]}$, etc. Besides their numerous practical applications, AUVs present a challenging control problem since the kinematic dynamic models of AUV are highly nonlinear and coupled.

Recently, the control of the underwater glider has become an active research field and the various control techniques have been reported for the control of the underwater glider ${ }^{[3-5]}$. For example, an optimal applicable control project is developed for controlling the actual mechanical system of the glider ${ }^{[3]}$. Feedback control is introduced for the glider motion robust of the autonomous underwater gliders ${ }^{[4]}$. In addition, the synchronization is important for the underwater glider. For example, it is critical in sampling water columns, mapping the whole aquatic environment and seeking pollutant sources. Precise synchronization is very challenging due to dynamic nonlinearity and strong coupling among multiple control inputs. Despite that several scholars ${ }^{[6-7]}$ have investigated the synchronization of general nonlinear system, it should be worth noting that these studies do not focus on the underwater glider. Therefore, it is still important and meaningful to design a novel controller for the synchronization of the underwater glider.

Inspired by the above-mentioned work, in this paper, we investigate the synchronization between the known master underwater glider and the unknown slave underwater glider.
The main difficulty to achieve this goal is how to overcome the bad effects of little knowledge about the slave underwater glider on the synchronization. An adaptive fuzzy sliding mode controller is designed to cope with the above difficulty and construct the synchronization of the unknown slave underwater glider.

The rest of this paper is organized as follows. The dynamic equation of an underwater glider is represented in Section II. Synchronization of an underwater glider is investigated theoretically and numerically by using the adaptive fuzzy sliding mode control method in Section III. Finally in Section VI, the conclusions are given.

\section{SYNCHRONIZATION OF UNDERWATER GLIDER}

\section{A. Dynamic Equation of Underwater Glider}

The dynamical equation of an underwater glider can be described as ${ }^{[8]}$

$$
\left\{\begin{array}{l}
\dot{x}_{1}=-\frac{X_{u}}{m_{11}} x_{1}-\left(\frac{X_{u|u|}}{m_{11}} x_{1}\left|x_{1}\right|-\frac{m_{22}}{m_{11}} x_{2} x_{3}\right) \\
\dot{x_{2}}=-\frac{Y_{v}}{m_{22}} x_{2}-\left(\frac{Y_{v|v|}}{m_{22}} x_{2}\left|x_{2}\right|+\frac{m_{11}}{m_{22}} x_{1} x_{3}\right) \\
\dot{x_{3}}=-\frac{N_{r}}{m_{33}} x_{3}-\left(\frac{N_{r|r|} x_{3}\left|x_{3}\right|}{m_{33}}-\frac{m_{11}-m_{22}}{m_{33}} x_{1} x_{2}\right)
\end{array}\right.
$$

where $m_{11}=m-X_{u}, m_{22}=m-Y_{v}, m_{33}=I_{z}-N_{r}$ In this paper, the values of parameters in the system (1) are respectively set as

$$
\begin{aligned}
& X_{u}=70 \mathrm{~kg} / \mathrm{s}, X_{u|u|}=100 \mathrm{~kg} / \mathrm{m}, Y_{v}=100 \mathrm{~kg} / \mathrm{s}, \\
& Y_{v|v|}=200 \mathrm{~kg} / \mathrm{m}, N_{r}=50 \mathrm{kgm}^{2} / \mathrm{s}, N_{r|r|}=100 \mathrm{kgm}^{2}, \\
& \text { so } m_{11}=215 \mathrm{~kg}, m_{22}=265 \mathrm{~kg}, m_{33}=80 \mathrm{kgm}^{2} .
\end{aligned}
$$

For the concision of the contents and the convenience of readers, we define the following parameters 
and

$$
\left\{\begin{array}{l}
f_{1}(\mathbf{x}, t)=-\frac{X_{u}}{m_{11}} x_{1}-\left(\frac{X_{u|u|}}{m_{11}} x_{1}\left|x_{1}\right|-\frac{m_{22}}{m_{11}} x_{2} x_{3}\right) \\
f_{2}(\mathbf{x}, t)=-\frac{Y_{v}}{m_{22}} x_{2}-\left(\frac{Y_{v|r|}}{m_{22}} x_{2}\left|x_{2}\right|+\frac{m_{11}}{m_{22}} x_{1} x_{3}\right) \\
f_{3}(\mathbf{x}, t)=-\frac{N_{r}}{m_{33}} x_{3}-\left(\frac{N_{r|r|} x_{3}\left|x_{3}\right|}{m_{33}}-\frac{m_{11}-m_{22}}{m_{33}} x_{1} x_{2}\right)
\end{array}\right.
$$

$$
f=\left[f_{1}(\mathbf{x}, t), f_{2}(\mathbf{x}, t), f_{3}(\mathbf{x}, t)\right]^{T} \in R^{3 \times 1}
$$

where $\boldsymbol{x}=\left(x_{1}, x_{2}, \cdots, x_{n}\right)^{T}$.

So Eq. (1) can be rewritten as

$$
\dot{x}=f(x, t)
$$

\section{B. Theoretical Results of Synchronization}

Let us define the master underwater glider as (2), and define the slave underwater glider as follow

$$
\dot{y}=g(y, t)+b u+d \quad y \in R^{n \times 1}
$$

where $\boldsymbol{u}=\left(u_{1}, u_{2}, \cdots, u_{n}\right)^{T} \in R^{n \times 1}$ is a control input. $\operatorname{diag}(\cdot)$ is a diagonal function. $\boldsymbol{d}=\left(d_{1}, d_{2}, \cdots, d_{n}\right)^{T} \in R^{n \times 1}$ is the external disturbance. It is assumed that $\left|d_{i}\right| \leq \bar{d}<+\infty$ for all $t$, where $\bar{d}$ is not necessarily known previously. $\boldsymbol{y}=\left(y_{1}, y_{2}, \cdots, y_{n}\right)^{T} \in R^{n \times 1}$ is the state vector of the slave system. $\boldsymbol{g}(\boldsymbol{y}, t)=\left[g_{1}(\boldsymbol{y}, t), g_{2}(\boldsymbol{y}, t), \cdots, g_{n}(\boldsymbol{y}, t)\right]^{T} \in R^{n \times 1}$ and

$$
\left\{\begin{array}{l}
g_{1}(\boldsymbol{y}, t)=-\frac{X_{u}}{m_{11}} y_{1}-\left(\frac{X_{u|u|}}{m_{11}} y_{1}\left|y_{1}\right|-\frac{m_{22}}{m_{11}} y_{2} y_{3}\right) \\
g_{2}(\boldsymbol{y}, t)=-\frac{Y_{v}}{m_{22}} y_{2}-\left(\frac{Y_{v|| \mid}}{m_{22}} y_{2}\left|y_{2}\right|+\frac{m_{11}}{m_{22}} y_{1} y_{3}\right) \\
g_{3}(\boldsymbol{y}, t)=-\frac{N_{r}}{m_{33}} y_{3}-\left(\frac{N_{r|r|} y_{3}\left|y_{3}\right|}{m_{33}}-\frac{m_{11}-m_{22}}{m_{33}} y_{1} y_{2}\right)
\end{array}\right.
$$

The synchronization error between (2) and (3) is defined as follow

$$
\boldsymbol{e}=\boldsymbol{y}-\boldsymbol{x}
$$

where $\boldsymbol{e}=\left(e_{1}, e_{2}, \cdots, e_{n}\right)^{T} \in R^{n \times 1}$.

Then, from (4), we can obtain the error dynamic equation as following

$$
\dot{e}=g(y, t)-f(x, t)+u+d
$$

The object of this paper is that for the given master-slave system with uncertainties, such as (2) and (3), the adaptive fuzzy sliding mode controllers are designed in spite of external disturbances, so that the asymptotic stability of system (5) can be achieved in the sense that

$$
\lim _{t \rightarrow+\infty} e=0 \quad \text { and } \quad \lim _{t \rightarrow+\infty} \dot{e}=0
$$

The switching sliding surface is defined as $s_{i}(t)=c_{i} e_{i}(t) \quad(i=1,2,3)$, where $c_{i}$ is a positive constant. Then the first derivative of $S_{i}(t)$ with respect to time $t$ is

$$
\dot{s}_{i}(t)=c_{i}\left[g_{i}(\boldsymbol{y}, t)-\lambda_{i} f_{i}(\boldsymbol{x}, t)+u_{i}+d_{i}\right] \quad(i=1,2,3)
$$

It is generally known that when the system operates in the sliding mode, it satisfies the following equations ${ }^{[9]}$

$$
s_{i}(t)=0 \quad \text { and } \quad \dot{s}_{i}(t)=0 \quad(i=1,2,3)
$$

Therefore, the equivalent controller is

$$
u_{i}=b_{i}^{-1}\left(-g_{i}+f_{i}-d_{i}\right) \quad(i=1,2, \cdots, n)
$$

However, in real-world applications, if both the system (3) is unknown, the equivalent controller (9) is failure. To control and identify the system (3), the fuzzy systems $\hat{g}_{i}(\boldsymbol{y}, t)$ and $\hat{d}_{i}$ are used to approach the functions $g_{i}(\boldsymbol{y}, t)$ and $d_{i}$, respectively. In this paper, the fuzzy rules of the fuzzy systems are defined as follows

$\mathrm{R}(\mathrm{j})$ : If $y_{i}$ is $A_{1}^{(j)}$ and $D_{t}^{q_{i}} y_{i}$ is $A_{2}^{(j)}$,then $\hat{g}_{i}$ is $G^{(j)}$;

$$
\text { If } s_{i} \text { is } C^{(j)} \text {, then } \hat{h}_{i} \text { is } H^{(j)} \text {. }
$$

Based on the singleton fuzzifier, the product inference, and the weighted average defuzzifier ${ }^{[10]}$, the fuzzy systems $\hat{g}_{i}\left(y_{i}, D_{t}^{q_{i}} y_{i}\right)$ and $\hat{h}_{i}\left(s_{i}\right)$ are respectively described by

$$
\hat{g}_{i}=\boldsymbol{\alpha}_{i}^{T} \boldsymbol{\xi}\left(y_{i}, \dot{y}_{i}\right) \text { and } \hat{h}_{i}=\boldsymbol{\gamma}_{i}^{T} \boldsymbol{\phi}\left(s_{i}\right)
$$

where $\boldsymbol{\alpha}_{i}$ and $\boldsymbol{\gamma}_{i}$ vary with the designed adaptive update laws. Both $\xi(\cdot)$ and $\phi(\cdot)$ are the fuzzy vectors and defined respectively as follows

$$
\boldsymbol{\xi}\left(y_{i}, \dot{y}_{i}\right)=\left(\xi^{(1)}\left(y_{i}, \dot{y}_{i}\right), \xi^{(2)}\left(y_{i}, \dot{y}_{i}\right), \cdots, \xi^{(N)}\left(y_{i}, \dot{y}_{i}\right)\right)^{T}
$$




$$
\phi\left(s_{i}\right)=\left(\phi^{(1)}\left(s_{i}\right), \phi^{(2)}\left(s_{i}\right), \cdots, \phi^{(N)}\left(s_{i}\right)\right)^{T},
$$

where $\quad \xi^{(j)}\left(y_{i}, \dot{y}_{i}\right)=\frac{\mu_{A_{i}^{(j)}}\left(y_{i}\right) \mu_{A_{2}^{(j)}}\left(\dot{y}_{i}\right)}{\sum_{j=1}^{N} \mu_{A_{i}^{(j)}}\left(y_{i}\right) \mu_{A_{2}^{(j)}}\left(\dot{y}_{i}\right)}$

and

$$
\phi^{(j)}\left(s_{i}\right)=\frac{\varphi_{C^{(j)}}\left(s_{i}\right)}{\sum_{j=1}^{N} \varphi_{C^{(j)}}\left(s_{i}\right)}
$$

$\mu_{A_{1}^{(j)}}\left(y_{i}\right), \mu_{A_{2}^{(j)}}\left(\dot{y}_{i}\right)$ and $\varphi_{C^{(j)}}\left(s_{i}\right)$ are respectively the membership functions of $y_{i}, \dot{y}_{i}$ and $s_{i} . N$ is the number of the fuzzy rules.

Thus, according to the linear feedback method, the controller is redesigned as following

$$
u_{i}=\hat{b}_{i}^{-1}\left(-\hat{g}_{i}+f_{i}-\hat{h}_{i}\right) \quad(i=1,2,3)
$$

To describe the new design and analysis, the following optimization parameters are needed

$$
\begin{gathered}
\boldsymbol{\alpha}_{i}^{*}=\arg \min _{\boldsymbol{\alpha}_{i} \in \Omega_{g}}\left[\sup \mid \hat{g}_{i}\left[\left(y_{i}, D_{t}^{q_{i}} y_{i}\right) \mid \boldsymbol{\alpha}_{i}\right]-g(\boldsymbol{y}, t)\right] \\
\gamma_{i}^{*}=\arg \min _{\gamma_{i} \in \Omega_{\gamma}}\left[\sup \mid \hat{h}_{i}\left(s_{i} \mid \gamma_{i}\right)-(\bar{d}+\delta) \operatorname{sgn}\left(s_{i}\right)\right]
\end{gathered}
$$

where $i=1,2, \cdots, n . \Omega_{g}$ and $\Omega_{\gamma}$ are the sets of $\boldsymbol{\alpha}_{i}$ and $\gamma_{i}$, respectively, and $\delta$ is a small positive number and $\operatorname{sgn}(\cdot)$ is a symbol function.

The minimum approaching error is defined as follow $w_{i}=g_{i}-\hat{g}_{i}^{*}$, where $\hat{g}_{i}^{*}=\hat{g}_{i}\left[\left(y_{i}, \dot{y}_{i}\right) \mid \alpha_{i}^{*}\right]$. Then Eq. (7) can be rewritten as

$$
\dot{s_{i}}=g_{i}-f_{i}+u_{i}+d_{i}=w_{i}+\tilde{\alpha}_{i}^{T} \boldsymbol{\xi}\left(y_{i}, \dot{y}_{i}\right)+\tilde{\gamma}_{i}^{T} \phi\left(s_{i}\right)-\hat{h}_{i}^{*}+d_{i}
$$

where $\tilde{\alpha}_{i}=\alpha_{i}^{*}-\alpha_{i}$ and $\tilde{\gamma}_{i}=\hat{\gamma}_{i}^{*}-\gamma_{i}$.

Theorem: When $g_{i}(\boldsymbol{y}, t)$ and $d_{i}$ are unknown, and $\hat{h}_{i}\left(s_{i} \mid \gamma_{i}^{*}\right)=(\bar{d}+\delta) \operatorname{sgn}\left(s_{i}\right)(\delta \geq 0)$

$s_{i} \in R$

$\left|d_{i}\right| \leq \bar{d}(i=1,2, \cdots, n)$, if the controllers are designed as
(10) and the adaptive update laws satisfy the following equations

$$
\left\{\begin{array}{l}
\dot{\boldsymbol{\alpha}}_{i}=r_{i} s_{i} \xi\left(y_{i}, D_{t}^{q_{i}} y_{i}\right) \\
\dot{\gamma}_{i}=\rho_{i} s_{i} \phi\left(s_{i}\right)
\end{array}\right.
$$

then systems (2) will synchronize systems (3).

Proof:

Construct a Lyapunov error function of the following

$$
V=\sum_{i=1}^{n} V_{i}
$$

where

$$
V_{i}=s_{i}^{2}+\frac{1}{2 r_{i}} \tilde{\boldsymbol{\alpha}}_{i}^{T} \tilde{\boldsymbol{\alpha}}_{i}+\frac{1}{2 \rho_{i}} \tilde{\gamma}_{i}^{T} \tilde{\gamma}_{i}
$$

Then the first derivative $V_{i}$ of with respect to time $t$ is

$$
\begin{aligned}
\dot{V}_{i}= & s_{i} w_{i}+\tilde{\boldsymbol{\alpha}}_{i}^{T}\left[s_{i} \boldsymbol{\xi}\left(y_{i}, \dot{y}_{i}\right)-\frac{1}{r} \dot{\boldsymbol{\alpha}}_{i}\right] \\
& +\tilde{\gamma}_{i}^{T}\left[s_{i} \phi\left(s_{i}\right)-\frac{1}{q_{i}} \dot{\gamma}_{i}\right]-\left(\bar{d}_{i}+\delta\right)\left|s_{i}\right|+d_{i} s_{i}
\end{aligned}
$$

where $\dot{\tilde{\alpha}}_{i}=-\dot{\boldsymbol{\alpha}}_{i}$ and $\dot{\tilde{\gamma}}_{i}=-\dot{\gamma}_{i}$.

Substituting Eq.(12) into Eq.(15), we yield the equation

$$
\dot{V}_{i}=s_{i} w_{i}-\left(\bar{d}_{i}+\delta\right)\left|s_{i}\right|+d_{i} s_{i}
$$

According to a fuzzy approaching theory ${ }^{[11]}$, an adaptive update fuzzy system can accurately approach any unknown system, so the minimum approaching error $w_{i}$ can approach zero.

$$
\text { So } \quad \dot{V}_{i} \leq s_{i} w_{i}+\left(\left|d_{i}\right|-\bar{d}_{i}-\delta\right)\left|s_{i}\right|<0
$$

then $\dot{V}=\sum_{i=1}^{n} \dot{V}_{i} \leq 0$

So $\dot{V} \leq 0$ confirms the presence of the reaching condition ${ }^{[12]}$, and means that $s_{i}$ is toward the switching surface $s_{i}=0 \quad(i=1,2, \cdots, n)$. Furthermore, since the sliding modes are asymptotically stable, it clearly shows that the error dynamic responses of $\lim _{t \rightarrow+\infty} \boldsymbol{e}=0$ and $\lim _{t \rightarrow+\infty} D_{t}^{q} \boldsymbol{e}=0$ are satisfied. Hence, the proof is achieved completely. 


\section{Numerical Simulation Results of Synchronization}

In this section, the numerical examples about the synchronization of an underwater glider will be provided. When numerically solving the equation of an underwater glider, we adopt the fourth order Runge-Kutta method ${ }^{[13]}$. $\alpha_{i}$ is defined as $25 \times 1$ vectors, and $\gamma_{i}$ is defined as $3 \times 1$ vectors, the initial values of $\boldsymbol{\alpha}_{i}$ and $\boldsymbol{\gamma}_{i}$ are set as 0.1 . The initial conditions of the master underwater glider and the slave underwater glider are selected as $\left(x_{1}(0), x_{2}(0), x_{3}(0)\right)=(-0.3,0.5,0.6)$ and $\left(y_{1}(0), y_{2}(0), y_{3}(0)\right)=(0.1,-0.1,-0.5) \quad, \quad$ respectively. $\left(c_{1}, c_{2}, c_{3}\right)=(2,5.5,4)$. The coefficients of the adaptive update laws are set as $\left(r_{1}, r_{2}, r_{3}\right)=(2,10,30),\left(p_{1}, p_{2}, p_{3}\right)=(1000,200,30)$ and $\left(\rho_{1}, \rho_{2}, \rho_{3}\right)=(1000,200,80)$, respectively.

Five kinds of membership degree functions are used to haze the errors variables $y_{i}, y_{i}$ and $s_{i}$ respectively defined as follows

$$
\begin{aligned}
& \mu_{N M}(z)=\exp \left[-\left(z+\frac{\pi}{6}\right)^{2} / \frac{\pi^{2}}{24^{2}}\right], \mu_{N S}(z)=\exp \left[-\left(z+\frac{\pi}{12}\right)^{2} / \frac{\pi^{2}}{24^{2}}\right] \\
& \mu_{Z O}(z)=\exp \left[-z^{2} / \frac{\pi^{2}}{24^{2}}\right], \mu_{P S}(z)=\exp \left[-\left(z-\frac{\pi}{12}\right)^{2} / \frac{\pi^{2}}{24^{2}}\right] \\
& \text { and } \mu_{P M}(z)=\exp \left[-\left(z-\frac{\pi}{6}\right)^{2} / \frac{\pi^{2}}{24^{2}}\right] .
\end{aligned}
$$

The membership degree functions of the switching sliding surface $s_{i}(i=1,2,3)$ are defined as follows

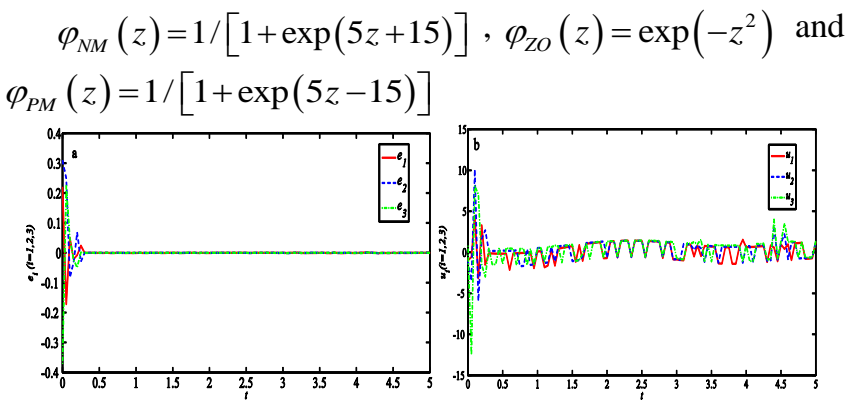

Figure 1. With external disturbances: (a) the temporal evolution of synchronization errors; (b) the temporal evolution of synchronization errors.

To verify and demonstrate the robustness of the controlled system for the external disturbances, the timevarying external disturbances (16) are used to disturb the unknown slave system. The synchronization errors between the known master underwater glider and the unknown slave underwater glider are depicted in figure 1(a). By observing Fig.1(a), one can find that the synchronization errors are hardly affected by the external disturbances and converge to zero asymptotically, which implies that the synchronization between the known underwater glider and the unknown underwater glider can be well realized even though the unknown slave underwater glider is subject to the uncertain external disturbances. The temporal evolution of the applied control inputs is depicted in figure 1(b). Therefore, the designed controllers (9) and (11) can resist all kinds of external disturbances and the synchronization is well achieved.

\section{CONCLUSION}

In this paper, we have studied the synchronization of the underwater glider, where the slave underwater glider is not necessarily known previously. Based on the designed adaptive update laws, the fuzzy system and the sliding mode strategy have been combined into an adaptive fuzzy sliding mode control strategy. To illustrate the effectiveness and feasibility of the proposed method, the numerical examples about the synchronization of an underwater glider are provided. The proposed scheme in this paper is remarkable, since it is valid even though the slave underwater glider is fully unknown. Therefore, the results of this paper are also very applicable and representative. We hope our work can contribute to the control of the underwater glider.

\section{ACKNOWLEDGMENT}

The authors are very grateful to the reviewers for their valuable comments and suggestions, which have led to the improved presentation of this paper. This work was partially supported by the Projects of Department of Science and Technology in Hebei Province, China (Grant No.15213401), the Research Foundation of Education Bureau of Hebei Province, China (Grant No.QN2014096) and the Langfang Teachers University Foundation, China (Grant No. LSLZ201404).

\section{REFERENCES}

[1] F. E. Farris, W. M. Rand, "Underwater glider (Patent style)", U.S. Patent 3157 145, November 17, 1964

[2] Rudnick D L, Davis R E, Eriksen C C, et al., "Underwater gliders for ocean research". Marine Technology Society Journal, 2004, 38(2): 73-84.

[3] S. W. Zhang, J. C. Yu, A. Q Zhang, "Optimal control for underwater gliders in the vertical plane", Control Theory \& Applications, 2012, 29(1): 19-26,

[4] N. E. Leonard, J. G. Grave, "Model-based feedback control of autonomous underwater gliders", Oceanic Engineering, IEEE Journal of, 2001, 26(4): 633-645

[5] S. Yuan, Y. Zheng, J. Wang, Model predictive control of underwater gliders based on a one-layer recurrent neural network[C]//Advanced Computational Intelligence (ICACI), 2013 Sixth International Conference on. IEEE, 2013: 328-333.

[6] Huang L, Feng R, Wang M, "Synchronization of chaotic systems via nonlinear control”, Physics Letters A, 2004, 320(4): 271-275.

[7] C. W. Wu, T. Yang, L. O. Chua, "On adaptive synchronization and control of nonlinear dynamical systems", International Journal of Bifurcation and Chaos, 1996, 6(3): 455-471.

[8] W. HOU, S. X. Wang, H. G. Zhang, "Dynamic modeling and control for miniature autonomous underwater vehicle", Journal of Tianjin University, 2004, 37(9): 769-773.

[9] J. K. Liu 2005 Intelligence control ( Bei Jing: Publishing House of Electronic Industory ) (in Chinese) 
[10] Y. Li, Y. Q. Chen, I. Podlubny,"Stability of fractional-order nonlinear dynamic systems: Lyapunov direct method and generalized MittagLeffler stability”, Comput. Math. Appl., vol.59, 2010, pp.1810

[11] T. Toshir, A. Kiyoji, S. Michio S, 1992 Fuzzy Systems Theory and Its Applications ( Boston: Academic Press )
[12] J. J. E. Slotine, W. P. Li, 1991 Applied Nonlinear Control (Englewood Cliffs: Prentice-Hall )

[13] M. J. Berger, Methods in computational physics. Academic, New York, 1963, 1: 135. 